\title{
Navigating our days in a culture of distraction
}

Tony Horava, Collection Coordinator, University of Ottawa: thorava@uottawa.ca

\begin{abstract}
:
In the past decade or so, librarians' working lives have been transformed by digital communication and information technologies. This has created an environment where distraction has become a normative state. We need to be cognizant of the impacts of distraction on our effectiveness. As library professionals working with information for a multiplicity of purposes, how do we adapt in ways that respect our human limitations? What are the implications of working in a state of continual distraction, and what strategies can we use to minimize this reality? This article reviews some of our daily distractions and draws associations from the literature in cognitive psychology and neuroscience to highlight the problems and raise potential solutions.
\end{abstract}

\section{Keywords:}

Information overload; distraction; memory; workplace; job performance

Lately I've been thinking a lot about information and memory. The distractions we face in our day to day library work are legion. We arrive in our office and the phone is flashing. We turn on the computer and the inbox pours forth its endless flow of messages, as we start scanning for urgent issues and sorting priorities in our mind. A colleague knocks at the door and wants to discuss an upcoming meeting. There are print memos pinned to the corkboard next to the door, and snail mail still arrives every day. The red light flashing on the telephone doggedly indicates that messages are waiting, and there are fax machines delivering information to us that needs to be actioned. Sound familiar? If not, you are likely in the minority. We spend our days in a culture of distraction - a veritable tsunami of information washing over us. It is a microcosm of the wider networked society of bits and bytes ricocheting in diverse formats and with almost inconceivable speed. One of the hallmarks of this culture is the frequency and constancy of distraction, and our habituated coping styles.

In a few short years, this has revolutionized our work, specifically, and our profession in general. We live in a multi-tasking, fragmented environment; and we work in a knowledge-based, service-oriented profession. We are information rich and time poor; it is the scarcity of time that determines how much we can absorb and process in a given day. This is ubiquitous in knowledge-based professions and occupations - librarianship is but one example. The maze of issues and the massive cognitive input facing us on a daily basis can lead to discouragement or alternatively to an adrenalin rush, but what are the implications for how we function, and what strategies can we use to make life 
more manageable for ourselves? In short, what are the biological limitations of the human brain to absorb and process information, and to make rational, intelligent decisions in a situation of frequent distraction?

The literature on cognitive psychology and neuroscience takes this issue quite seriously. Perusing the cited literature on http://Interruptions.net (Interruptions in Human-Computer Interaction) gives a sampling of the wide research in this field from engineers to neuroscientists to software developers. It's an intriguing and different lens through which to perceive our digital age and its effects on task performance and efficiency. Erik Altmann, in a piece entitled 'Managing Attention by Preparing to Forget', conducted research on the memory-updating problem using a serial attention paradigm involving simple operational tasks. He concluded: "A qualitative prediction of our analysis is that human operators in dynamic task environments need time for encoding and decay --without time to pay adequate attention to an update, and without enough time to let an item fade, situation awareness could degrade catastrophically'" (155). I often doubt that we have enough time to pay adequate attention to information in order to ensure encoding and avoid neurological decay! Altmann was investigating simple, quick, repetitive tasks, rather than the complex tasks and issues we face in libraries. I imagine that this analysis has an even stronger resonance for us- we need adequate time for information to be encoded before moving on to the next matter requiring our attention.

It's easy to forget how in just a few short years, the pace and tenor of our working lives has radically changed. Compare our work environment to say, fifteen or twenty years ago, and the contrast is quite stark. The speed of information delivery and the overlapping multiplicity of issues and events couldn't have been imagined in those days. Communication in those days was incremental and predictable; today it is exponential and wholly unpredictable. Apart from the ubiquitous inbox for new mail, there are alerting services that zap us with announcements; RSS feeds that regularly push information to us; calendars that inform us of meetings; there are devices such as Blackberries or PDAs that are ever ready to stream messages; and cell phones that vibrate or ring. How many interruptions do we face every hour, every day? I've never deliberately tried to count, but I would assume it would be a significant number, by nature of the multi-channel wired world in which we work. And is this changing our consciousness in ways we don't fully comprehend? There is no doubt that the brain of a professional librarian in 2008 needs to function in rapid, cognitive ways that are very different from the pre-Internet world of twenty years ago.

And of course, there is the ubiquitous web browser that takes us in a hundred directions, and every time we discover an important new page or blog, there is another virtual door that beckons us to try our luck, and so we click on another link. I've heard this described as 'flicking or skimming behaviour', and it fits in with our environment - we 
all do this as a strategy of survival. It is clear that mental association of ideas while web exploring will often lead us to new avenues of investigation for analysis, thus creating more channels with more information. We are now working in an accelerated environment of cognitive overflow that is constantly punctuated by a barrage of interruptions -- both internal and external - thus making sustained concentration difficult. This also has a major impact on the formation of long-term memory. John Lorinc, in his article 'Driven to Distraction', addresses this issue. He relates a description of memory by Frank Russo, an associate professor of psychology at Ryerson University:

"In most models of working memory and attention, everything has to go through a central executive processor before being passed into long-term memory...... Our built-in CPUs are found in the brain's frontal lobe. These centres need time to "rehearse" or "scaffold" incoming information by building the neural circuits on which the data will eventually be stored. "If it is not rehearsed enough or elaborated upon"..."the information will never make it to the long-term store" (50).

I expect that all of us are beset with situations where there isn't time for the brain's frontal lobes to be engaged in this fashion, and thus we lose the ability to store everything in long-term memory for future recall. Frequently I seem to be rummaging in the back of my consciousness, trying without success to remember a particular event, fact, or circumstance. I see this occurring in colleagues as well, as the daily barrage of information we have to filter and absorb is ill-fitted for the 'central executive processor', that cerebral matter we depend upon for our ability to function competently. While we may joke about premature aging, the reality is that our technology is making it difficult for our brains to function in their customary biological manner, and we don't yet know the long-term implications for memory and cognition. Lorinc writes that, "we must acknowledge the self-inflicted memory lapses triggered by information overload, chronic interruptions, and relentless electronic multi-tasking" (59). It is no small irony that we, whose fundamental role is to acquire, organize, preserve and access information for our user community, are struggling with the problems of managing the information tsunami in our own workspace. This adds a whole new layer of complexity to our work, and I imagine that guilt and anxiety are never far from the surface when we find ourselves beset with these challenges. I'd be surprised if any of us never or rarely experience these emotions!

We live at the dawn of a heady and bewildering new era of digital communication, but this is undermined by the taxing demands of information technology, and we need to find a balance. Over the next few generations it will be interesting to see if the evolutionary nimbleness of the brain and its adaptability for survival will lead to structural changes in how we process and segment information for reuse and recall in other contexts. Philip Agre points to one of the contradictions of our times: "The irony is that one of the fundamental promises of information technology -- the radical 
improvement in the efficiency of our interactions with one another -- is being undermined by the technology's enormous capacity to overwhelm us with information and thus short-circuit our need to concentrate"(13).

Electronic multi-tasking has become a way of life for many of us, as we try to juggle the demands of email, phone calls, report writing, blog monitoring, and the imperious demands of various electronic devices. It isn't possible, however, for the brain to focus with equal attention on two simultaneous tasks, and our effectiveness is bound to suffer as one of the tasks gets shorter shrift. We work in a highly technological space where separating relevant information from noise is a continuous task that requires energy and judgment. Think of all the obvious junk mail and not so obvious junk mail that needs to be digested; think of everything you see and read in the course of a day that requires you to judge its value for retention. To file or delete, that is the question. As digital communication technology inexorably rushes onward, it is clear that the flow of information in all directions will only increase. Philip Agre notes that, "As the culture digests these technologies, people will invent new always-on cultural forms that depend on each party to a relationship having access to large amounts of continually updated information about the other"(13).

As our environment will continue to consist of data feeds that remain 'always-on', and social relationships develop in new (and virtual) directions, it's clear that we need to find strategies for sifting the wheat from the chaff and using our abilities in the most productive and sanest ways possible. Here are a few ideas that come to mind: 1) Schedule blocks of time in your calendar for work that requires sustained concentration (if you can); 2) Resist the urge to check your inbox constantly; instead figure out at what intervals during the day it makes the most sense to do so; 3) If you have a Blackberry, use it responsibly and don't let it become an addiction; 4) Establish priorities and goals for each day and stick to your program unless urgent issues require you to do otherwise; 5) Find clues or markers when scanning email to quickly determine whether an item can be deleted, filed, or needs to be actioned; 6) Be realistic in your expectations of what can be achieved in a day, given the expected and unexpected distractions that will occur; and 7) Learn to expect the occasional moment of forgetfulness!

While these ideas may be useful in and of themselves, the challenge occurs in managing the expectations of colleagues who may have different strategies and expectations -- and pressures or deadlines originating from other sources. The fine art of interpersonal communication and diplomatic negotiation is the best remedy in these circumstances. If we can communicate our challenges proactively and effectively with our colleagues, we can alleviate our situation to a certain degree. Much depends on the workplace climate. 
The blurring of the professional and the personal that occurs today is another sign of the times -- this weakening or erasure of boundaries is the consequence of ubiquitous information technologies in our lives. As the medium of communication is frequently the same, it can happen so easily that we don't even notice until it becomes a problem. How we negotiate with technology, in particular communication devices and office productivity tools will have an impact on how effective we can be in the digital era. The metaphor of navigation is routinely applied to the Web, but it applies equally well to the routines and activities in our hectic working lives. Maintaining the skill of being able to focus on an issue in a sustained manner is so important in today's environment. Distraction is a cultural reality in our work, but it's important to be aware of the limitations of being human and the pitfalls of our 'always-on' world, and how this impacts our thinking, decision-making, and effectiveness. The balance between push and pull is such a critical equation for our sanity! Equal dollops of humour and humility can help guide us as well. We teach our users how to navigate and evaluate the sea of information resources, but we need to navigate our own spaces, both mental and physical, with a zen-like awareness and judicious care, in order to maintain our own professional balance.

\section{Works Cited}

Agre, Philip. "Welcome to the Always-On World." IEEE Spectrum 38:1, (2001): 10-13.

Altmann, Erik et al. "Managing Attention by Preparing to Forget" Proceedings of the IEA 2000/HFES 2000 Congress. Santa Monica: Human Factors and Ergonomics Society, 2000. 152-155.

Lorinc, John. "Driven to Distraction." The Walrus 4:5, (2007): 50-59. 